Check for updates

Cite this: RSC Adv., 2017, 7, 20212

\title{
Identification and quantification of immunoglobulin G (G1, G2, G3 and G4) in human blood plasma by high-resolution quadrupole- Orbitrap mass spectrometry
}

\begin{abstract}
Zhu Huang*ab and Xiao-Dong Pan (DD *c
We described a method for quantification of $\lg G$ (IgG1, IgG2, $\lg G 3$ and $\lg G 4$ ) in a small amount of human blood plasma using high-resolution quadrupole-Orbitrap mass spectrometry (HR-Q-Orbitrap-MS). For screening surrogate peptides of targeted IgGs, samples were prepared by pellet digestion and identified by data-independent acquisition of Orbitrap MS. The quantification was performed by parallel reaction monitoring (PRM). The remarkable sensitivity and selectivity of PRM enable the detection of low abundance IgGs, or a little plasma. The assay linearity for external standard IgG proteins was obtained from 5 to $1000 \mu \mathrm{g} \mathrm{L}^{-1}$ with a mean coefficient of determination $\left(R^{2}\right)$ higher than 0.99 . The inter-day $(n=$ 6) and intra-day $(n=6)$ precisions obtained were from $3.6 \%$ to $11.5 \%$. This report represents a comprehensive study so far of the use of Q-Orbitrap-MS for the identification and quantitation of IgGs in human blood plasma.
\end{abstract}

Received 3rd March 2017 Accepted 2nd April 2017

DOI: 10.1039/c7ra02623d

rsc.li/rsc-advances

\section{Introduction}

Profiling of immunoglobulins with modern instruments such as liquid chromatography (LC) and mass spectrometry (MS) is of great interest to researchers. ${ }^{1}$ The antibody repertoire has the potential to hold a library detailing an individual's antigen exposure history, which may reveal key information about individual's immune system. In the clinical lab, monitoring of Ig antigen specificity has brought to light immunoglobulin biomarkers for evaluation on immunity, autoimmunity and cancer detection. $^{2-5}$

Conventional methods for detecting clinically significant Igs are based on antigen specificity. Enzyme-linked immunosorbent assay (ELISA) and cell/tissue-based immunofluorescence (IF) have been the main methods for detecting Ig biomarkers. ${ }^{1}$ The basic principle of these methods is the detection of Igantigen complexes formed by the interaction of a patient's serum with a known antigen.

One new approach with LC-MS/MS to the detection of specific antibodies has begun to emerge in the literature., ${ }^{\mathbf{1 , 6 - 8}}$ Unlike the current approaches based on Ig-antigen interaction, it seeks to further probe fundamental structure information of

${ }^{a}$ Department of Ophthalmology, The First Affiliated Hospital, College of Medicine, Zhejiang University, Hangzhou, China.E-mail: zhuzhu2003@126.com; Tel: +86571 87115274

${ }^{b}$ Department of Ophthalmology, The Forth Affiliated Hospital, College of Medicine, Zhejiang University, Yiwu, China

${ }^{c}$ Zhejiang Provincial Center for Disease Control and Prevention, Hangzhou, China. E-mail: zjupanxiaodong@hotmail.com; Tel: +8657187115274 the Ig protein. The most commonly LC-MS/MS method is to digest the targeted protein in samples and screen one or more peptides with good selectivity and sensitivity as surrogate analyte of the protein. The majority of quantitative assays were achieved by LC systems hyphenated to triple quadrupole (QqQ) mass analyzers operating in mode of selected reaction monitoring (SRM).$^{9,10}$ For example, Hong et al. ${ }^{11}$ and Ladwig et al. ${ }^{12}$ demonstrated that IgG glycoforms can also be quantified with subclassspecific proteotypic peptides by use of LC-MS/MS. However, interferences in mass response from other generated tryptic peptides in complex matrices (serum or plasma) at the retention time of the surrogate peptide(s) of interest may not be excluded, even though appropriate sample cleanup strategies such as solid phase extraction (SPE) can reduce the sample complexity. ${ }^{13}$

To improve the method selectivity for quantitative analysis, application of high resolution mass spectrometry (HRMS) such as quadrupole time of flight (QTOF), Orbitrap or Fourier transform ion cyclotron resonance mass analyzer is a promising way. ${ }^{14-16}$ On contrast to three-quadrupole MS (TQ-MS), one of the main advantages of HRMS is the high mass accuracy less than $5.0 \mathrm{ppm}$ as well as the highly resolved isotopic pattern with a resolution ( $>25000)$.

Recently, the use of HRMS for the quantification of small molecules and larger peptides in human plasma was successfully demonstrated. ${ }^{17-19}$ Sun et $a .^{20}$ demonstrated that QExactive is proper for proteomic analysis in terms of obtained peptide and protein group. Mekhssian et $a .^{21}$ reported a method for antibody quantification by a LC system coupled to a TripleTOF ${ }^{\mathrm{TM}} 5600$ mass spectrometry. 
The present study aims to explore the capabilities of UPLC-QOrbitrap-MS (Q-Exactive) for the identification and quantification of immunoglobulin G (IgG1, G2, G3 and G4) in human blood plasma. The development of a UPLC-Q-Orbitrap-MS method for the quantification of IgGs in plasma is described using pellet digestion as sample preparation combined with data-dependent acquisition for surrogate peptides detection and PRM.

\section{Materials and methods}

\section{Chemicals}

Acetonitrile, methanol, formic and acetic acid were all purchased from Merck (Darmstadt, Germany). Sodium acetate, magnesium sulphate and ammonium formate were purchased from Sigma-Aldrich. Standard IgG (1-4) from human plasma (purified immunoglobulin, >95\%) were purchased from Sigma. For the calibration of the MS, the Pierce LTQ Velos ESI positiveion calibration solutions (Thermo Fisher Scientific, Waltham, MA, USA) were used.

\section{Chromatographic \& MS conditions}

A Vanquish UHPLC system consisting of a quaternary pump, an autosampler and a column oven was used in this test (all Thermo Scientific, San Jose, CA, USA). Chromatographic separation was performed on a $2.1 \times 50 \mathrm{~mm}, 1.7 \mu \mathrm{m}$, BEH300 $\mathrm{C}_{18}$ column (Waters Corporation, MA, USA) with a $0.5 \mu \mathrm{m}$ frit. The column temperature was set at $60^{\circ} \mathrm{C}$. Mobile phase A consisted of $0.1 \%$ formic acid in water, and mobile phase B consisted of $0.1 \%$ formic acid in acetonitrile. Samples were separated by gradient elution using the following program: 0 min $3 \% \mathrm{~B}$; 0$0.8 \min 3 \% \mathrm{~B}$; 0.8-5 min 60\% B; 5-6 min 100\% B; 6-7 min $100 \%$ B; $8-10 \mathrm{~min} 3 \% \mathrm{~B}$. The flow rate was $0.3 \mathrm{ml} \mathrm{min}^{-1}$. The sample injection volume was $5 \mu \mathrm{L}$.

The UPLC system was coupled to a Q-Orbitrap-MS equipped with a heated electrospray ionization probe (HESI II) operating in positive mode (Thermo Fisher Scientific). Nitrogen was used for spray stabilization, for collision-induced dissociation experiments in the higher energy collision dissociation (HCD) cell, and as the damping gas in the C-trap. The following ionization parameters were applied: electrospray voltage $3.0 \mathrm{kV}$ for positive mode, capillary temperature $350{ }^{\circ} \mathrm{C}$, vaporizer temperature $250{ }^{\circ} \mathrm{C}$, sheath gas $\left(\mathrm{N}_{2}\right) 40$ arbitrary units (arb), auxiliary gas $\left(\mathrm{N}_{2}\right) 15$ (arb), and S-Lens RF level at 50 (arb). The instrument was calibrated in positive mode every 7 days using the Pierce LTQ Velos ESI positive-ion calibration solutions from manufacturer (containing caffeine, the tetrapeptide MRFA and a mixture of fluorinated phosphazines ultramark 1621).

Two different scan modes, full MS/dd-MS ${ }^{2}$ for peptide identification and PRM for quantification, were performed in this study. For a full MS scan, the selected scan range was from $\mathrm{m} / z 100$ to 1500 and the resolution was 35000 (FWHM at $\mathrm{m} / \mathrm{z}$ 200), while the automatic gain control (AGC) target (the number of ions to fill C-Trap) was set to $1.0 \times 10^{6}$ with a maximum injection time (IT) of $100 \mathrm{~ms}$. For the dd-MS ${ }^{2}$ scan, the fragmentation mass spectra were recorded at a mass resolving power of $17500 \mathrm{FWHM}$ with a quadrupole isolation window of $0.4 \mathrm{Da}$ for precursor ions. Other MS parameters for the dd-MS ${ }^{2}$ scan were applied as follows: AGC target $2.0 \times 10^{5}$, maximum IT $50 \mathrm{~ms}$, underfill ratio $1.0 \%$, intensity threshold $4.0 \times 10^{4}$, exclude isotopes 'on', and dynamic exclusion $10.0 \mathrm{~s}$. For PRM scan, the parameters were: default charge 2, inclusion on, $\mathrm{MS}^{2}$ resolution 17500 , maximum IT $100 \mathrm{~ms}$, AGC target $2.0 \times 10^{5}$, isolation window $4.0 \mathrm{~m} / z$, and NCE/stepped $20,35,55$. The accurate masses for the precursor are shown in Table 1.

\section{Sample preparation}

All experiments on the human blood were approved by Human Research Ethics Committee of the First Affiliated Hospital, College of Medicine, Zhejiang University and performed in accordance with the relevant guidelines. We confirmed that informed consent was obtained from all subjects for usage of human blood. Human plasma samples were collected from author of Xiao-Dong Pan. Plasma samples $(25 \mu \mathrm{L})$ were transferred to Eppendorf tube prior to sample preparation. Methanol $(75 \mu \mathrm{L})$ was added, and followed by vortex mixing at $1000 \times g$ for $1 \mathrm{~min}$. After centrifuge at $4000 \times g$ for $5 \mathrm{~min}$ at $5{ }^{\circ} \mathrm{C}$, the supernatants were carefully discarded. Pellets were resuspended by addition of $100 \mu \mathrm{L} \mathrm{NH}_{4} \mathrm{HCO}_{3}(500 \mathrm{mM})$ and 720 $\mu \mathrm{L}$ deionized water followed by vortexing at $2000 \times g$ for $10 \mathrm{~min}$. A $20 \mu \mathrm{L}$ aliquot of $50 \mathrm{mM}$ DTT solution were added to the mixtures and reduced in $40{ }^{\circ} \mathrm{C}$ air bath for $30 \mathrm{~min}$ at this stage. In the next step, an alkylation was performed by adding $20 \mu \mathrm{L}$ of $150 \mathrm{mM}$ IAA in the dark for $30 \mathrm{~min}$ at room temperature. Pellet digestion was carried out by addition of $100 \mu \mathrm{L}$ of $500 \mathrm{mM}$ $\mathrm{NH}_{4} \mathrm{HCO}_{3}$ and $25 \mu \mathrm{L}$ of $1 \mathrm{mg} \mathrm{mL}{ }^{-1}$ trypsin (freshly prepared) were added and incubated $6 \mathrm{~h}$ at $37{ }^{\circ} \mathrm{C}$. The reaction was terminated by addition of $5 \mu \mathrm{L}$ formic acid. Prior to injection onto the UPLC-Q-Orbitrap-MS system, the digested sample plates were centrifuged at $6000 \times g$ for $5 \mathrm{~min}$ at $5{ }^{\circ} \mathrm{C}$ to remove pellet particulates.

\section{UPLC-Q-Orbitrap-MS data acquisition, processing \& sample quantification}

The system was controlled using the software packages Xcalibur 3.0, Chromeleon MS Link 2.14 and Q-Exactive Tune 2.3 (all Thermo Scientific). Xcalibur software was used for data acquisition and processing. For identification analytical runs, full MS/dd-MS ${ }^{2}$ scan was adopted. The obtained data were treated by the software of Proteome Discoverer ${ }^{\mathrm{TM}} 2.1$ (Thermo Fisher Scientific, Waltham, MA, USA) for peptide confirmation and screening. For quantitative analytical runs, PRM scan were performed with the precursor and fragment ions. The calibration curve was performed using peak area of standard proteins with different concentrations. Duplicate 6 point calibration standards, placed at the beginning and end of each analytical run, were fitted to linear regression model. The equation of this curve was then used to calculate the predicted concentrations in all study samples within the analytical runs. 
Table 1 Information of identified peptides of IgGs by data-dependent acquisition of HR-Q-Orbitrap-MS ${ }^{a}$

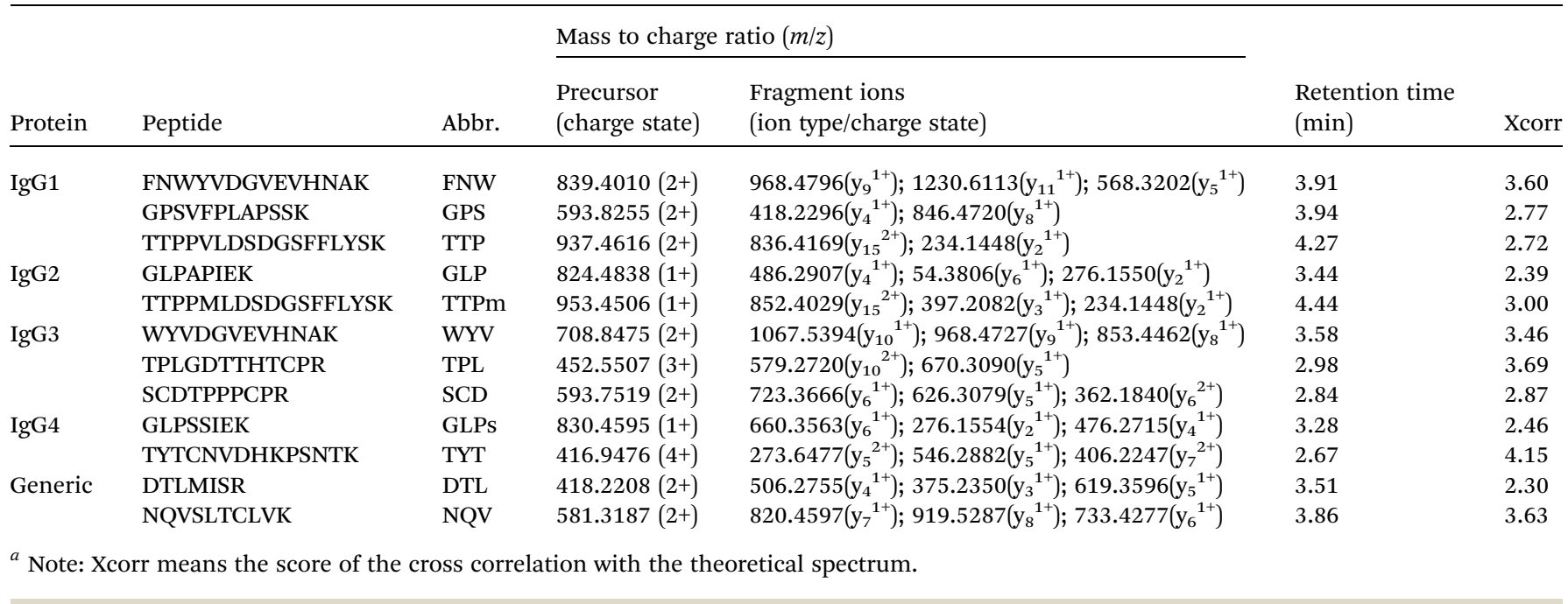

\section{Results and discussion}

We developed a method for the absolute quantitation of immunoglobulin G (G1-4) in human plasma using Q-OrbitrapMS with PRM. The workflow of the method is depicted in Fig. 1. Human IgG standards were used for absolute quantification by external calibration method. Before quantification test, IgG standards were tryptic digested and analyzed by full scan/dd$\mathrm{MS}^{2}$ mode of Q-Orbitrap MS for obtaining the surrogate peptides and fragment ions. These peptides and their fragments were carefully screened based on the criteria of selectivity, stability and sensitivity. Selected signal peptides and their fragments were used for PRM quantification analysis. Surrogate peptides for targeted IgGs were obtained by pellet digestion which might reduce the matrix effect of mass response. ${ }^{22,23}$ The method was further validated for the specificity, sensitivity, accuracy and precision.

\section{Identification of signature peptides by full scan/dd-MS ${ }^{2}$ analysis}

Besides the light and heavy chain, the structure of antibodies can be further subdivided into variable and constant regions. The latter is only suitable for a universal MS-based assay in pre-

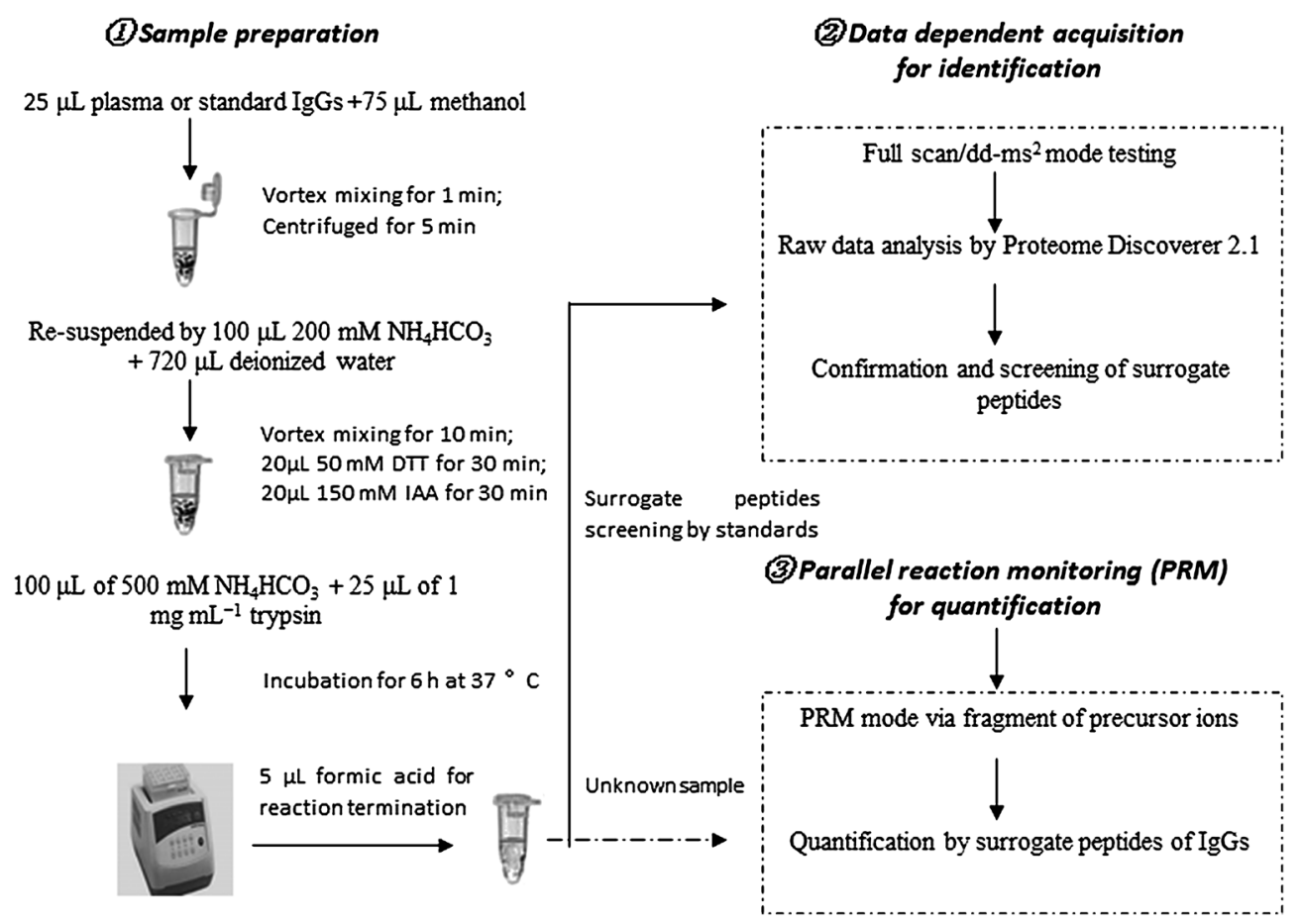

Fig. 1 The workflow of IgGs identification and quantification by HR-Q-Orbitrap-MS. 
clinical species as the majority of the amino acid sequence is conserved over all human antibodies exhibiting common peptides after tryptic digestion, whereas the former contains antibody specific peptides located in the complementarity determining region being responsible for specific target binding. For the identification of conserved peptides, the amino acid sequences of the Fc region from IgG were aligned with each other and an in silico digestion was performed. Furthermore, the mixed IgG standards were tryptic digested and tested by mode of full scan/dd-MS ${ }^{2}$ which is data-dependent acquisition based on HCD (Fig. 2).

Raw data were then processed through the commercial software Proteome Discoverer ${ }^{\mathrm{TM}} 2.1$ and protein assignment was accomplished via Sequest scoring algorithm by searching QOrbitrap-MS against human immunoglobulin database (downloaded from http://www.uniprot.org/ on Jan. 10, 2016). The number and sequence of the identified peptides were retrieved for each allergenic protein family by interrogating the data bank at a high confidence and a false discovery rate (FDR) set at 0.01 (indicating a measure of certainty in the identification). According to the criteria that signature peptides are unique to the target protein and detectable by the MS systems of choice, a summary of detected targeted peptides is schematized in Table 1. The MS/ MS product ion spectra of identified surrogate peptides are shown in Fig. 3. Furthermore, peptides showing Q-Orbitrap-MS spectra highly matching with the predicted fragmentation patterns and displaying the highest Xcorr were further analysed by the analyst by visual inspection of the generated spectra in order to select those showing the highest intensity and reproducibility in the same state charge (variance $\leq 15 \%$ ) and the best chromatographic resolution from matrix interferences.

\section{Screening of surrogate peptides}

The criteria followed for peptide markers detection were: (1) reproducibility of the retention time $\leq 0.1 \mathrm{~min}$, (2) mass accuracy better than $\leq 5 \mathrm{ppm}$, (3) at least two fragment ions detected in the high resolution MS, and (4) relatively high intense of mass response. So, the targeted peptides were screened and listed (Table 1), and those peptides with low mass accuracy and mass intensity were not chosen.

Furthermore, prepared samples (spiked with standard IgGs) were analyzed again after 20 days storage at $-20,-4,10$, and $37{ }^{\circ} \mathrm{C}$ separately for peptide stability test. As shown in Fig. 4, there were no obvious changes in percentage of intensity to original after 20 days storage at $-20{ }^{\circ} \mathrm{C}$. With the raising of temperature, the intensities of selected peptides were decreased. According to these changes, we adopt FNW for IgG1, GLP for IgG2, WYV for IgG3, GLP for IgG4 and DTL as general surrogate peptide for all IgGs. The data of peptide stability indicated that the storage of digested samples for Q-OrbitrapMS analysis should be kept at as low temperature as possible.

\section{IgG quantitation using PRM}

From the fragmentation patterns obtained from full scan/dd$\mathrm{MS}^{2}$ analysis, PRM transitions were developed for the peptides. The common or general surrogate peptide to all four types and selected for quantitation is DTLMISR. Its PRM transition from precursor ion $\left([\mathrm{M}+2 \mathrm{H}]^{2+} \mathrm{m} / z\right.$ 418.22) to fragment ion $\mathrm{m} / z$ 506.28 and $m / z 375.23$ was selected as the quantifier. The optimized fragmentation voltage was determined to be $9 \mathrm{eV}$ for the quantifier. For other surrogated peptides, the following transitions were determined to be optimal: $\left([\mathrm{M}+2 \mathrm{H}]^{2+} 839.40 \rightarrow \mathrm{m} / \mathrm{z}\right.$ 968.48 and $m / z$ 1230.61) for IgG1, $\left([\mathrm{M}+3 \mathrm{H}]^{3+} 824.48 \rightarrow \mathrm{m} / \mathrm{z}\right.$ 486.29 and $m / z 54.38)$ for IgG2 , and $\left([\mathrm{M}+3 \mathrm{H}]^{3+} 708.85 \rightarrow \mathrm{m} / z\right.$ 1067.54 and $m / z$ 968.47) for IgG3, $\left([\mathrm{M}+3 \mathrm{H}]^{3+} 830.46 \rightarrow \mathrm{m} / z\right.$ 660.36 and $m / z 276.16$ ) for IgG4. The PRM transitions for all peptides, together with their respective fragmentation voltages are listed in Table 1. Previous study also reported that generic peptide of DTLMISR can be used for IgG quantification by LCQTOF-MS with multiple reaction monitoring (MRM). ${ }^{11}$

The PRM technique has several potential advantages over MRM or SRM approach. ${ }^{24,25}$ First, PRM spectra would be highly specific because all potential product ions of a peptide, instead of just 3-5 transitions, are available to confirm the identity of the peptide. ${ }^{26,27}$ Second, PRM could provide a higher tolerance for coisolated background peptides/species. Because numerous ions

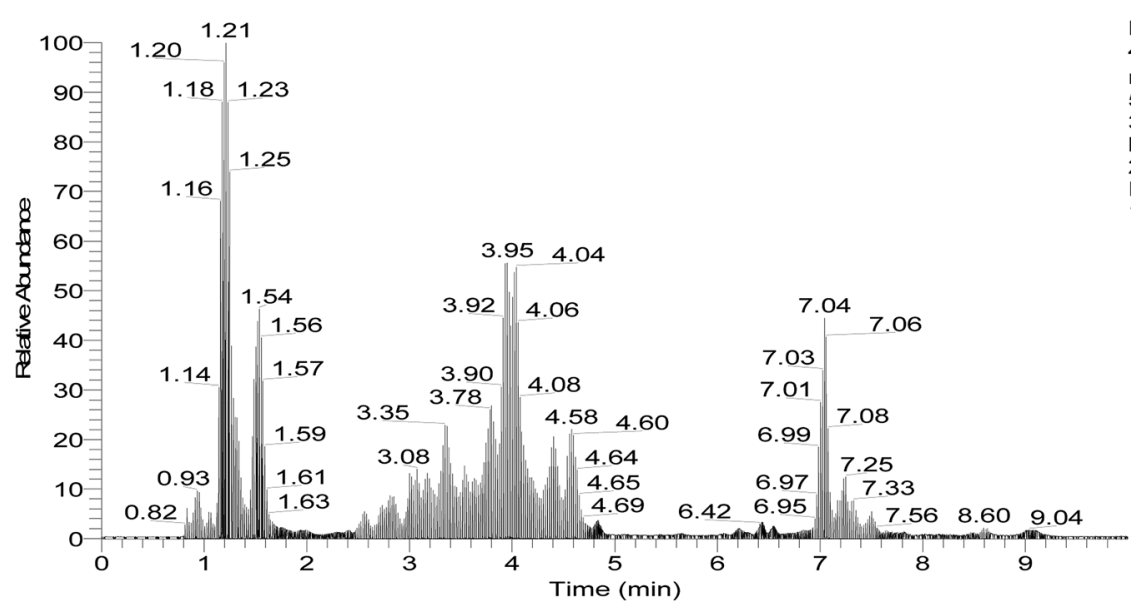

NL:

4.11E9

$\mathrm{m} / \mathrm{z}=$

$50.00000-$

3430.00000

MS

20160524-

$1 \mathrm{Gg}-\mathrm{sd}$-ddms-

Fig. 2 Chromatograms of peptides in blood plasma by data-dependent acquisition of UPLC-Q-Orbitrap-MS (full scan/dd-MS ${ }^{2}$ ). 

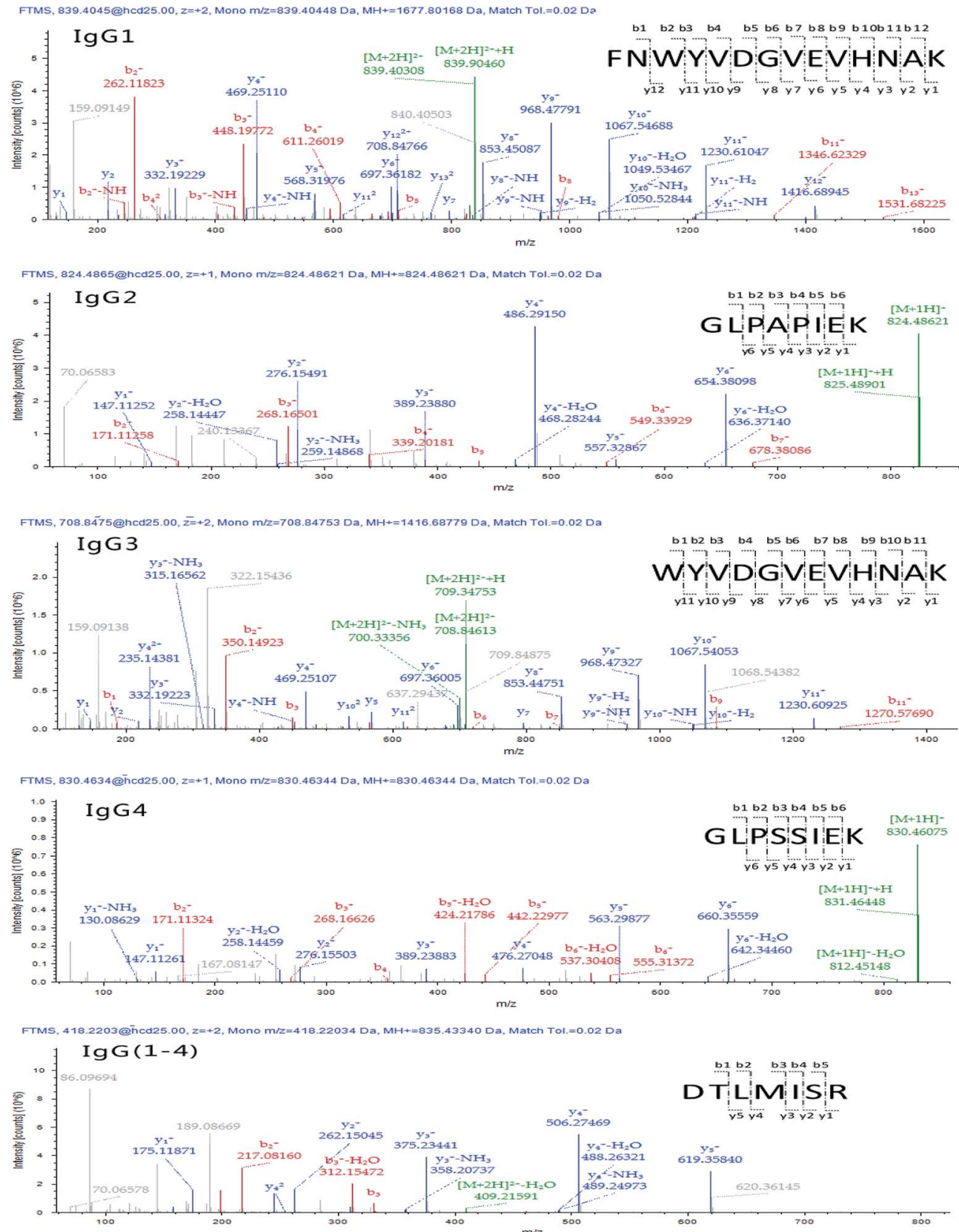

Fig. $3 \mathrm{MS} / \mathrm{MS}$ product ion spectra of surrogate peptides for IgGs.

would be available for identification and quantitation purposes, the presence of interfering ions in a full mass spectrum would be less disruptive to overall spectral quality than interference in a narrow mass range, especially because high resolution can often separate these ions from the product of interest. ${ }^{28}$

\section{Method validation}

Specificity. The sequence specificity of surrogate peptides FNW for IgG1, GLP for IgG2, WYV for IgG3, GLP for IgG4 and DTL as general peptide for all IgGs were confirmed by the results of online BLAST search in UniProt (http:// www.uniprot.org). The chromatograms of the selected peptide from tryptic serum sample spiked with standard IgGs showed the sharp and symmetric peaks without interferences. Furthermore, there was not target peak appeared in the serum sample without tryptic digestion. All these results indicated that the selected surrogate peptides had a high specificity for quantitation of IgGs in human serum.

Linearity and sensitivity. The linear regression equations for the concentration sequences that ranged from 5 to $1000 \mu \mathrm{g} \mathrm{L} \mathrm{L}^{-1}$ are listed in Table 2 with good linearity and coefficient of determination $\left(R^{2}>0.99\right)$. The LODs calculated as the lowest concentration that provided a signal-to-noise ratio of 3 were $2.5 \mu \mathrm{g} \mathrm{L}^{-1}$ for IgG1, 3.4 $\mu \mathrm{g} \mathrm{L}^{-1}$ for IgG2, 1.2 $\mu \mathrm{g} \mathrm{L}^{-1}$ for IgG3 and $4.0 \mu \mathrm{g} \mathrm{L}^{-1}$ for IgG4. For generic surrogate peptide DTL, the LOD of all IgGs was 


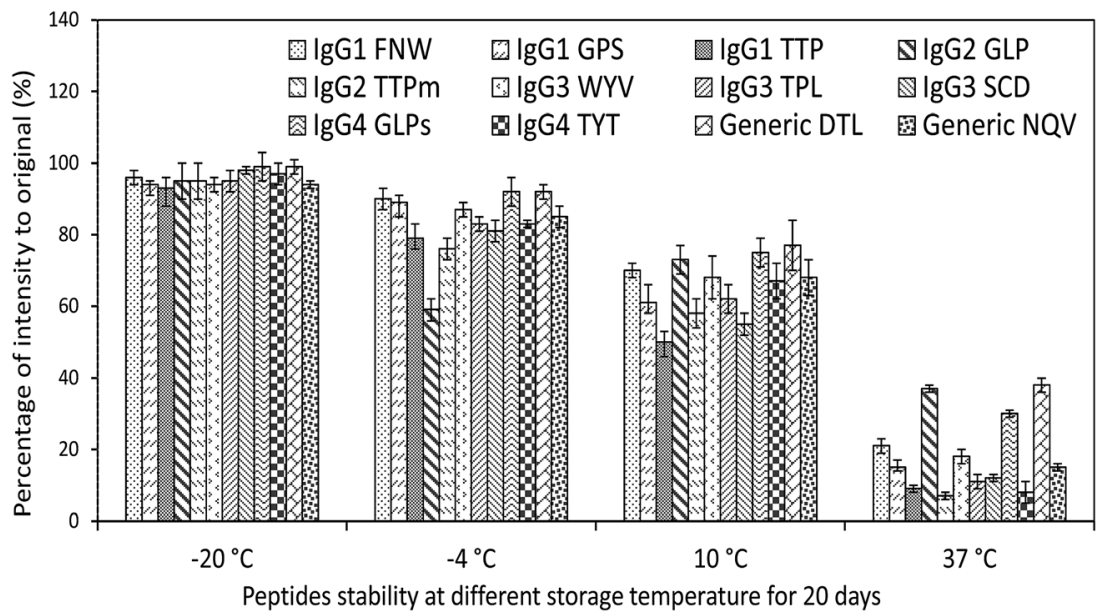

Fig. 4 The stability of selected peptides from IgGs during the storage with different temperatures.

Table 2 The precision, accuracy, LOD $\left(\mu \mathrm{g} \mathrm{L}^{-1}\right)$ and linearity of IgGs for quantification of PRM in HR-Q-Orbitrap-MS ${ }^{a}$

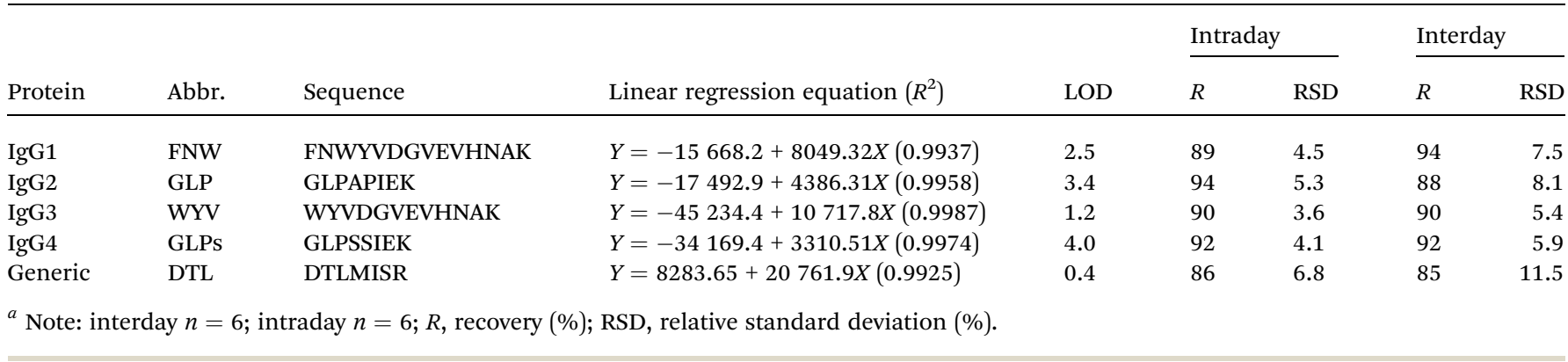

$0.4 \mu \mathrm{g} \mathrm{L}^{-1}$. The linear range and sensitivity could fully meet the quantification requirements of the different concentrations of IgGs in human serum. Comparing with previous report by LCQTOF-MS with MRM, ${ }^{11}$ LC-Q-Orbitrap-MS with PRM showed the higher sensitivity which had lower LODs less than $5 \mu \mathrm{g} \mathrm{L}^{-1}$.

Intra- and inter-day precision and accuracy. For validation of accuracy and precision, standard IgGs were spiked to the supernatants (proteins removed by methanol in step of sample preparation) at a final level of $50 \mu \mathrm{g} \mathrm{L}^{-1}$ for IgG1, IgG2, IgG3 and IgG4. To evaluate the intraday precision of the developed method, plasma sample was prepared and analyzed 6 times. The results of relative standard deviations (RSDs) are depicted in Table 2, which are all lower than $10 \%$. The inter-day repeatability was evaluated by analyzing the above sample (stored at $-20{ }^{\circ} \mathrm{C}$ ) once per day for a total period of 6 days. The results showed high inter-day repeatability (RSD $\leq 15 \%$ ). It should be noted that generic surrogate peptide of DTLMISR had a higher standard deviation ( $\mathrm{RSD}=11.5 \%)$ in inter-day test. It may be caused by the partial oxidation of methionine in preparation procedures. Intra- and inter-day average recoveries were ranged from $85 \%$ to $94 \%$ (Table 2 ).

\section{Conclusion}

We conducted a systematic evaluation of UPLC-Q-Orbitrap-MS for the identification and quantification of immunoglobulin
G (G1, G2, G3 and G4), one of the most abundant proteins in human blood plasma. It is observed that PRM is a powerful technique for the absolute quantitation of immunoglobulin G. The selectivity of high resolution mass spectrometry reduces the interference of sample matrix. The sensitivity of the PRM method allows detection of IgG at the $\mu \mathrm{g} \mathrm{L}^{-1}$ level. The method facilitates simultaneous determination of IgG1, IgG2, IgG3 and IgG4 protein with small amount of blood plasma (at least $25 \mu \mathrm{L})$.

\section{Author contributions statement}

X.-D. P. and Z. Huang conceived the experiment(s), X.-D. P., Z. H. conducted the experiment(s), X.-D. P. and Z. H. analyzed the results. All authors reviewed the manuscript.

\section{Conflicts of interest}

The authors declare no competing financial interests.

\section{Acknowledgements}

The authors are thankful to Y. R. Jiang, X. Ke, J. S. Zhang and Z. $\mathrm{X}$. Cai for the help on the experiment. 


\section{References}

1 D. Murray and D. Barnidge, Crit. Rev. Clin. Lab. Sci., 2013, 50, 91-102.

2 K. Conrad, D. Roggenbuck, D. Reinhold and U. Sack, Autoimmun. Rev., 2012, 11, 207-211.

3 P. V. Belousov, D. V. Kuprash, S. A. Nedospasov and Y. V. Shebzukhov, Curr. Mol. Med., 2010, 10, 115-122.

4 E. R. Stiehm, J. Immunotoxicol., 2008, 5, 227-234.

5 G. A. Maguire, D. S. Kumararatne and H. J. Joyce, Ann. Clin. Biochem., 2002, 39, 374-377.

6 I. Trbojevic-Akmacic, M. Vilaj and G. Lauc, Expert Rev. Proteomics, 2016, 13, 523-534.

7 C. Lanshoeft, T. Wolf, O. Heudi, S. Cianferani, S. Barteau, M. Walles, F. Picard and O. Kretz, Anal. Bioanal. Chem., 2016, 408, 1687-1699.

8 N. Kawaguchi-Sakita, K. Kaneshiro-Nakagawa, M. Kawashima, M. Sugimoto, M. Tokiwa, E. Suzuki, S. Kajihara, Y. Fujita, S. Iwamoto, K. Tanaka and M. Toi, Biochem. Biophys. Res. Commun., 2016, 469, 1140-1145.

9 S. Gallien, E. Duriez and B. Domon, J. Mass Spectrom., 2011, 46, 298-312.

10 D. Lebert, G. Picard, C. Beau-Larvor, L. Troncy, C. Lacheny, B. Maynadier, W. Low, N. Mouz, V. Brun, C. KlinguerHamour, M. Jaquinod and A. Beck, Bioanalysis, 2015, 7, 1237-1251.

11 Q. Hong, C. B. Lebrilla, S. Miyamoto and L. R. Ruhaak, Anal. Chem., 2013, 85, 8585-8593.

12 P. M. Ladwig, D. R. Barnidge, M. R. Snyder, J. A. Katzmann and D. L. Murray, Clin. Chem., 2014, 60, 1080-1088.

13 Q. Zhang, D. S. Spellman, Y. L. Song, B. Choi, N. G. Hatcher, D. Tomazela, M. Beaumont, M. Tabrizifard, D. Prabhavalkar,
W. Seghezzi, J. Harrelson and K. P. Bateman, Anal. Chem., 2014, 86, 8776-8784.

14 S. Gallien and B. Domon, Expert Rev. Proteomics, 2015, 12, 489-498.

15 S. Gallien and B. Domon, Methods, 2015, 81, 15-23.

16 S. Gallien, E. Duriez, K. Demeure and B. Domon, J. Proteomics, 2013, 81, 148-158.

17 L. Dillen, W. Cools, L. Vereyken, W. Lorreyne, T. Huybrechts, R. de Vries, H. Ghobarah and F. Cuyckens, Bioanalysis, 2012, 4, 565-579.

18 L. P. Morin, J. N. Mess and F. Garofolo, Bioanalysis, 2013, 5, 1181-1193.

19 S. Ramagiri and F. Garofolo, Bioanalysis, 2012, 4, 529-540.

20 L. Sun, G. Zhu and N. J. Dovichi, Rapid Commun. Mass Spectrom., 2013, 27, 157-162.

21 K. Mekhssian, J. N. Mess and F. Garofolo, Bioanalysis, 2014, 6, 1767-1779.

22 Z. Ouyang, M. T. Furlong, S. Wu, B. Sleczka, J. Tamura, H. Q. Wang, S. Suchard, A. Suns, T. Olah, A. Tymiak and M. Jemal, Bioanalysis, 2012, 4, 17-28.

23 C. Lanshoeft, O. Heudi and S. Cianferani, Anal. Biochem., 2016, 501, 23-25.

24 N. Rauniyar, Int. J. Mol. Sci., 2015, 16, 28566-28581.

25 A. Bourmaud, S. Gallien and B. Domon, Proteomics, 2016, 16, 2146-2159.

26 J. Sherman, M. J. McKay, K. Ashman and M. P. Molloy, Proteomics, 2009, 9, 1120-1123.

27 M. W. Duncan, A. L. Yergey and S. D. Patterson, Proteomics, 2009, 9, 1124-1127.

28 A. C. Peterson, J. D. Russell, D. J. Bailey, M. S. Westphall and J. J. Coon, Mol. Cell. Proteomics, 2012, 11, 1475-1488. 